\title{
Reduction of carrier mobility in semiconductors caused by charge-charge interactions
}

\author{
E. Hendry, ${ }^{1, *}$ M. Koeberg, ${ }^{2}$ J. Pijpers, ${ }^{2}$ and M. Bonn ${ }^{2}$ \\ ${ }^{1}$ School of Physics, University of Exeter, Stocker Road, Exeter EX4 4QL, United Kingdom \\ ${ }^{2}$ FOM Institute for Atomic and Molecular Physics, Kruislaan 407, 1098 SJ, Amsterdam, The Netherlands
}

(Received 8 April 2007; revised manuscript received 14 May 2007; published 13 June 2007)

\begin{abstract}
We investigate the effect of charge-charge interactions on carrier mobility in titanium dioxide $\left(\mathrm{TiO}_{2}\right)$ and silicon $(\mathrm{Si})$ using terahertz spectroscopy. Charge scattering times and plasma frequencies are directly determined as a function of charge density. In Si, a linear increase in scattering rate for densities exceeding $10^{21} \mathrm{~m}^{-3}$ is attributed to electron-hole scattering. In contrast, in $\mathrm{TiO}_{2}$, charge-charge interactions are suppressed due to dielectric screening, highlighting the vastly different dielectric properties for these two materials.
\end{abstract}

DOI: 10.1103/PhysRevB.75.233202

PACS number(s): 72.40.+w, 72.10.Di, 72.20.Dp

Charge-charge interactions determine many of the properties of highly doped or photoexcited semiconductors. Carrier-carrier collisions, for instance, can strongly affect the conductivity, and the decrease of electron and hole drift mobilities with increasing charge carrier densities is an important factor in determining the current-voltage characteristics of, for example, semiconductor diodes at increased current densities ${ }^{1}$ and solar cells under strong illumination conditions. $^{2}$

Charge mobility depends on two quantities: its effective mass and scattering time (i.e., the average time in-between momentum-randomizing events, due to, e.g., electronphonon scattering). Theory has indicated that both of these quantities can be strongly dependent on the free charge density in semiconductors. ${ }^{3-5}$ Experimental investigations of carrier-carrier interactions are, however, intrinsically more complex, as most experimental techniques (such as optical reflectivity measurements ${ }^{6,7}$ ) cannot easily distinguish between changes in scattering time and effective mass. Terahertz time-domain spectroscopy (THz-TDS), with its ability to determine both the real and imaginary components of the complex conductivity, ${ }^{8}$ allows us to differentiate between density-dependent changes in effective mass from changes in scattering time.

Here, we compare the high-density photoconductivity of single crystal silicon $(\mathrm{Si})$ and rutile-type titanium dioxide $\left(\mathrm{TiO}_{2}\right)$, two technologically relevant materials with low and high dielectric functions, respectively. By photoexciting with intense, femtosecond ultraviolet laser pulses and probing with $\mathrm{THz}$ pulses, we can generate and measure high-density plasmas in semiconductors on ultrafast time scales, accessing plasma densities spanning 5 orders of magnitude from $10^{21}$ to $10^{26} \mathrm{~m}^{-3}$. In $\mathrm{Si}$, we find that the scattering rate increases linearly for densities $>10^{21} \mathrm{~m}^{-3}$, an effect characteristic of direct charge-charge scattering. For $\mathrm{TiO}_{2}$, the variation of the scattering rate with density is much weaker and is caused by band filling effects. The difference between these two materials is shown to originate from their vastly different dielectric screening properties.

The $\mathrm{TiO}_{2}$ (1 mm thick 001 rutile crystal grown commercially by the Verneuil method by Crystal-GmbH) and silicon ( $0.5 \mathrm{~mm}$ thick undoped $\mathrm{Si}$ wafer, grown by the Czochralski method with estimated impurity density $<10^{13} \mathrm{~cm}^{-3}$, purchased from Virginia Semiconductor) samples are excited above band gap with $150 \mathrm{fs,} 266 \mathrm{~nm}$ laser pulses, allowing for the generation of high charge densities. The samples are cooled to $30 \mathrm{~K}$ in a helium cryostat to minimize effects of electron-optical phonon scattering that prevail at higher temperatures in $\mathrm{TiO}_{2}$.

The THz-TDS setup is similar to that described in Ref. 10. We extend previous THz-TDS conductivity measurements on doped ${ }^{11}$ and photoexcited semiconductors ${ }^{9,12}$ to study electron-hole plasmas in highly photoexcited semiconductors. We determine in the time domain the electric field of picosecond $\mathrm{THz}$ pulses transmitted through the unexcited sample $E(t)$, and the change on photoexcitation $\Delta E(t)$, as shown in Fig. 1. By varying the time in-between $266 \mathrm{~nm}$ pump and $\mathrm{THz}$ probe pulses, we initially observe a rise in the magnitude of $\Delta E$ (see inset of Fig. 1) which we attribute to cooling of the nascent hot electrons and holes to their respective band edges (which typically takes a few picoseconds ${ }^{13}$ ). Below, we present measurements of $\Delta E(t) 10$ ps after excitation-sufficiently long to allow for thermalization and cooling of hot charges but fast enough to minimize diffusive expansion of the plasma ${ }^{14}$ or carrier trapping and/or recombination, which occurs on nanosecond time scales.

The initial charge distribution is determined by the penetration depth $l_{0}$ of the excitation light $(\sim 20 \mathrm{~nm}$ for rutile and $\sim 10 \mathrm{~nm}$ for $\mathrm{Si}$ found from Refs. 15 and 16, respectively). Since $l_{0}$ is small compared to the THz wavelength, the system can be approximated as a thin slab of excited material embedded in a homogeneous material with dielectric function $\varepsilon\left(\sim 120\right.$ and $\sim 12$ for $\mathrm{TiO}_{2}$ and $\mathrm{Si}$, respectively). The frequency-dependent complex conductivity $\sigma(\omega)$ can be inferred from the Fourier transforms of the timedomain fields shown in Fig. 1 using $^{17}$

$$
\sigma^{a v}(\omega)=-\frac{2 \varepsilon_{0} c \sqrt{\varepsilon}}{l_{0}} \frac{\Delta E(\omega)}{E(\omega)},
$$

where $\varepsilon_{0}$ is the permittivity of a vacuum, $c$ is the speed of light, and $\sigma^{a v}(\omega)$ represents an averaged conductivity for all photocharges across the excited region. For both $\mathrm{TiO}_{2}$ (Ref. 9) and $\mathrm{Si}^{18}$ electrons dominate the $\mathrm{THz}$ response, being more mobile than holes.

The data presented below can be reproduced very well using the Drude expression $\sigma^{a v}(\omega)=\varepsilon_{0} \omega_{p}^{2} \tau^{a v} /\left(1-i \omega \tau^{a v}\right)$, with $\omega_{p}=\sqrt{ }\left[e^{2} N^{a v} /\left(\varepsilon_{0} m_{c o n}\right)\right]$, the plasma frequency, which 


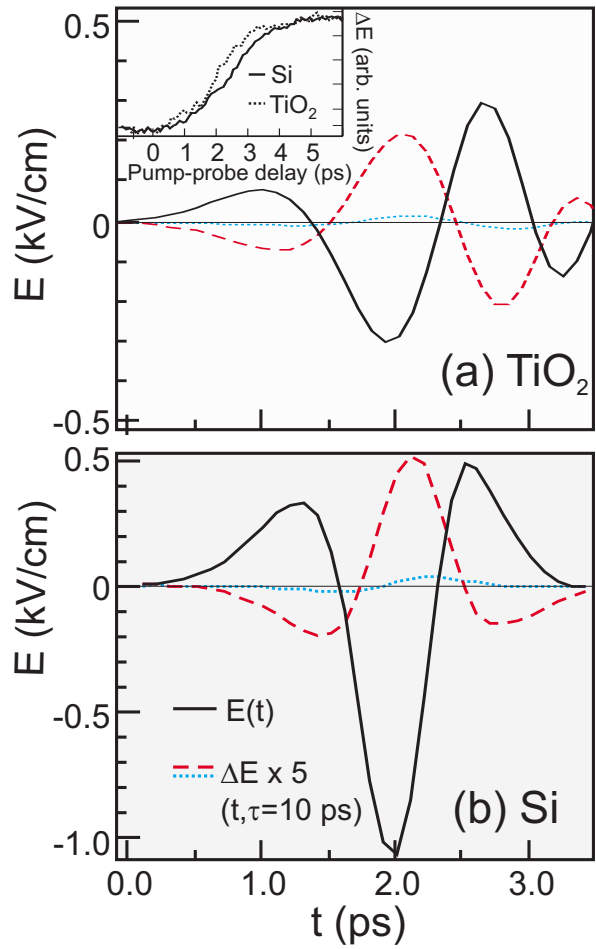

FIG. 1. (Color online) THz transmission $E$ and modulation $\Delta E$ (scaled by a factor of 5) $10 \mathrm{ps}$ after photoexcitation of (a) $\mathrm{TiO}_{2}$ and (b) Si with a $266 \mathrm{~nm}$ laser pulse. The red dashed and blue dotted lines are for high and low excitation fluences, respectively (values as stipulated in Fig. 2). Inset: $\Delta E$ at $t=2$ ps measured as a function of $266 \mathrm{~nm}$ pump-THz probe delay for $\mathrm{Si}$ and $\mathrm{TiO}_{2}$, scaled for comparison.

depends on the carrier density $N^{a v}$ (determined from the excitation pulse and the absorption spectrum ${ }^{19}$ ), the conductivity effective mass $m_{c o n}$, and scattering time $\tau^{a v}$. Since the density of charges is not homogeneous in the photoexcited region of the sample, ${ }^{19}$ we use the superscript " $a v$ " to indicate the average over this region. ${ }^{20}$

Figure 2 shows $\sigma^{a v}$, normalized to incident fluence, for $\mathrm{TiO}_{2}$ and $\mathrm{Si}$. For fluences $f_{l}<1 \mathrm{~J} / \mathrm{m}^{2}$ [Fig. 2(a) depicts $\sigma^{a v}(\omega)$ for $\left.f_{l} \sim 0.18 \mathrm{~J} / \mathrm{m}^{2}\right]$, the generated densities are sufficiently low that charges are uncorrelated and the frequency dependence of $\sigma^{a v}$ in $\mathrm{TiO}_{2}$ does not vary significantly. Plotted in Fig. 2(b) is the $\mathrm{TiO}_{2}$ conductivity observed for $f_{l}$ $\sim 6.40 \mathrm{~J} / \mathrm{m}^{2}\left(8.6 \times 10^{18}\right.$ photons $\left./ \mathrm{m}^{2}\right): \sigma^{a v}$ clearly changes significantly at higher density. A similar trend is observed for $\mathrm{Si}$ (lower panels in Fig. 2). The observed changes are not due to laser heating effects.

The different low-fluence conductivities for $\mathrm{TiO}_{2}$ and $\mathrm{Si}$ [Figs. 2(a) and 2(c)] can be fully accounted for by the differences in $\tau^{a v}$ of isolated carriers in these different materials: the Drude model (lines in Fig. 2) describes the data with parameters $\omega_{p}=38 \mathrm{THz}$ and $\tau^{a v}=0.4 \mathrm{ps}$ for $\mathrm{TiO}_{2}$ [lines in Fig. 2(a) $]$ and $\omega_{p}=95 \mathrm{THz}, \tau^{a v}=2.1 \mathrm{ps}$ for $\mathrm{Si}$ [lines in Fig. 2(c)]. The low plasma frequency in $\mathrm{TiO}_{2}$ (despite the approximately tenfold higher fluence compared to $\mathrm{Si}$ ) is caused by the high electron mass in this material; the smaller scattering time by more efficient coupling to phonons ${ }^{9}$ even at these low temperatures. Upon increasing $f_{l}$, both the scatter-

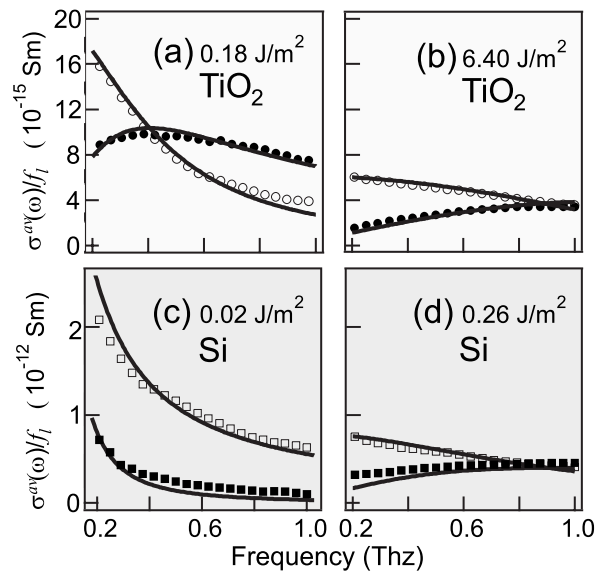

FIG. 2. Real (open symbols) and imaginary (filled symbols) of the complex conductivity for $\mathrm{TiO}_{2}$ (in the $\perp$ direction, upper panels) and $\mathrm{Si}$ (lower panels), measured with different $266 \mathrm{~nm}$ excitation fluences at $30 \mathrm{~K}$ : (a) $f_{l} \sim 0.18 \mathrm{~J} / \mathrm{m}^{2}, 2.4 \times 10^{17}$ photons $/ \mathrm{m}^{2}$; (b) $6.40 \mathrm{~J} / \mathrm{m}^{2}, \quad 8.6 \times 10^{18}$ photons $/ \mathrm{m}^{2}$; (c) $0.02 \mathrm{~J} / \mathrm{m}^{2}, \quad 2.5$ $\times 10^{16}$ photons $/ \mathrm{m}^{2}$; and (d) $0.26 \mathrm{~J} / \mathrm{m}^{2}, 3.5 \times 10^{17}$ photons $/ \mathrm{m}^{2}$. All conductivities are normalized by the incidence excitation fluence. Solid lines represent the Drude model.

ing rates and plasmon frequencies increase strongly: to reproduce the data in Figs. 2(b) and 2(d) requires $\omega_{p}$ $=220 \mathrm{THz}$ and $\tau^{a v}=0.18 \mathrm{ps}$ for $\mathrm{TiO}_{2}$ and $\omega_{p}=360 \mathrm{THz}, \tau^{a v}$ $=0.18 \mathrm{ps}$ for $\mathrm{Si}$.

Figure 3(a) shows that $\omega_{p}$ scales linearly with $f_{l}$ : the straight lines are fits assuming $\omega_{p}=\sqrt{ }\left[e^{2} N^{a v} /\left(\varepsilon_{0} m_{c o n}\right)\right]$, with $N^{a v}$ calculated from Ref. 19. The best fits occur for $m_{c o n}^{\mathrm{Si}}$ $=0.2 m_{e}$ and $m_{c o n}^{\mathrm{TiO}_{2}}=6 m_{e}$ ( $m_{e}$ being the free electron mass), comparable to previous reports of the low density, electron effective masses in Si (Ref. 21) and $\mathrm{TiO}_{2} \cdot{ }^{9}$ For $\mathrm{TiO}_{2}$, the deviation from linearity for $f_{l}>1 \mathrm{~J} / \mathrm{m}^{2}$ suggests that the effective mass in this material is density dependent: these deviations occur for $N^{a v}>1 \times 10^{25} \mathrm{~m}^{-3}\left(\omega_{p}>70 \mathrm{THz}\right){ }^{19}$ similar to zinc oxide. ${ }^{22}$ For $\mathrm{Si}$, band calculations ${ }^{3}$ indicate that the electron effective mass is constant over the density range investigated here, partly due to the sixfold degenerate band structure in Si.

The average scattering rate, meanwhile, increases dramatically in both materials upon increasing $f_{l}$, as shown in Fig. 3(b). The striking difference in fluence dependence between the two materials will be explained in the following.

In these undoped, single crystal samples, impurity scattering is expected to be negligibly small. Indeed, for $\mathrm{TiO}_{2}$, electron-phonon interactions have been shown to suffice to account for the temperature-dependent scattering rate down to $10 \mathrm{~K} .{ }^{9}$ For $\mathrm{Si}$, it has been shown that impurity scattering rates drop below phonon scattering rates as doping levels are decreased, also at $30 \mathrm{~K} .{ }^{23} \mathrm{We}$ therefore analyze our data as a sum of contributions from charge-phonon scattering $\left(1 / \tau_{p h}\right)$ and charge-charge scattering $\left(1 / \tau_{e h}\right)$. Both rates are affected at high carrier concentrations: to a first approximation, $1 / \tau_{e h}$ will increase proportionally to the charge concentration $N$, while, due to band filling, $1 / \tau_{p h}$ will scale as $N \frac{1}{3} .{ }^{24}$ The summed scattering rate may then be written as 




FIG. 3. (a) The plasma frequency $\omega_{p}$ as a function of excitation fluence $f_{l}$. The lines are straight line fits assuming $\omega_{p}^{2}$ $=e^{2} N^{a v} /\left(\varepsilon_{0} m_{\text {con }}\right)$, with $N^{a v}$ estimated from $f_{l}$ using Ref. 19. (b) Relaxation rate obtained from fitting the high-density conductivity after excitation with $266 \mathrm{~nm}$ pulses, plotted as a function of excitation fluence $f_{l}$. The lines are fits using Eq. (2). Note that for $\mathrm{TiO}_{2}$, the scattering time is almost density independent in the density range up to $10^{24} \mathrm{~m}^{-3}$.

$$
\frac{1}{\tau}=\frac{1}{\tau_{0}}+a N+b(N)^{1 / 3}, \quad b=\frac{\hbar}{l_{p h} m_{\mathrm{DOS}}}\left(\frac{3 \pi^{2}}{M}\right)^{1 / 3},
$$

where $1 / \tau_{0}$ is the scattering rate in the low-density limit and $a$ and $b$ (Ref. 24) are proportionality constants.

For $\mathrm{TiO}_{2}$, the photocharge density can be estimated directly from the fluence. ${ }^{14,19}$ The data in Fig. 3(b) are best described by Eq. (2) $[M=1$ (Ref. 25)] with $a=0$ [i.e., no charge-charge scattering, line in Fig. 3(a)] and $b=8$ $\times 10^{3} \mathrm{~ms}^{-1}$, though reasonable fits can be obtained with 0 $<a<2.3 \times 10^{-14} \mathrm{~m}^{3} \mathrm{~s}^{-1}$ and $4 \times 10^{3}<b<8 \times 10^{3} \mathrm{~m} \mathrm{~s}^{-1}$. This range of $b$ corresponds to an electron density of states effective mass $m_{\mathrm{DOS}} \sim 4 m_{e}-17 m_{e}$, consistent with calculated values of the conductivity effective mass $^{9}$ (note that we do not expect the density of states and conductivity effective masses to be identical as rutile is anisotropic). The results of this analysis show that the increase in scattering rate with increasing density in $\mathrm{TiO}_{2}$ is a result of enhanced electronphonon interactions caused by band filling rather than direct carrier-carrier scattering.

For $\mathrm{Si}$, in contrast, the linear dependence of the scattering rate on fluence in Fig. 3(b) suggests that charge-charge scattering effects dominate rather than band filling. One impor-

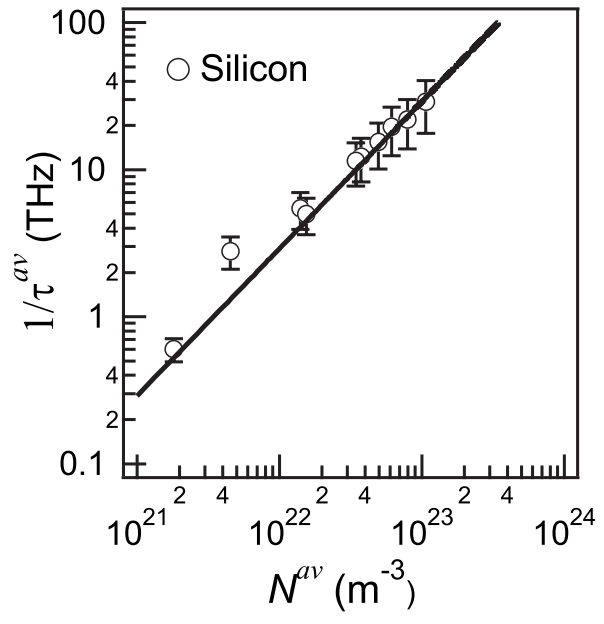

FIG. 4. Relaxation rate of carriers in silicon after excitation with $400 \mathrm{~nm}$ pulses, plotted vs. charge density (Ref. 19). The line is a fit using Eq. (2).

tant reason for the apparent absence of a contribution from enhanced electron-phonon scattering in $\mathrm{Si}$ lies in the band structure: while the band structure of $\mathrm{TiO}_{2}$ is nondegenerate, $\mathrm{Si}$ has a multivalley band minimum $[M=6$ in Eq. (2)], so that band filling effects will not be observed until a sixfold higher density is reached.

Due to charge diffusion in-between pump and probe pulses, ${ }^{14}$ a precise determination of the charge density in the Si sample is difficult when exciting with $266 \mathrm{~nm}$ pulses. In order to estimate more accurately the density at which charge-charge scattering becomes important in $\mathrm{Si}$, we repeat the measurements with $400 \mathrm{~nm}$ light excitation, for which the penetration depth is significantly larger $\left[l_{0} \sim 180 \mathrm{~nm}\right.$ (Ref. 16)]. This reduces the effects of charge diffusion between pump and probe pulses ${ }^{14}$ and allows for the quantitative determination of charge densities. ${ }^{19}$ In Fig. 4, we plot the scattering rate as a function of charge density. The line is the best fit using Eq. (2), corresponding to $a=2.9 \times 10^{-10} \mathrm{~m}^{3} / \mathrm{s}$. It is clear that the scattering rate is density dependent already at densities as low as $10^{21} \mathrm{~m}^{-3}$ in $\mathrm{Si}$, i.e., at densities more than 3 orders of magnitude lower compared to $\mathrm{TiO}_{2}$.

A notable discrepancy exists between this result $(a$ $\left.=2.9 \times 10^{-10} \mathrm{~m}^{3} \mathrm{~s}^{-1}\right)$ and theoretical predictions $[a \sim 0.8$ $\times 10^{-10} \mathrm{~m}^{3} \mathrm{~s}^{-1}$ (Refs. 4 and 5)]. As the drift momentum is conserved by collisions between particles of identical charge, one might presume that electron-hole scattering determines the rates plotted in Fig. $4,{ }^{6,7}$ but the anisotropic band structure of $\mathrm{Si}$ allows collisions between charges with the same sign, i.e., electron-electron and hole-hole scattering, to also contribute to drift momentum relaxation. At optical frequencies, Sernelius has suggested that this may be a very large effect, ${ }^{26}$ complicating a comparison between scattering theory and experiments at optical frequencies. ${ }^{7,26}$ In the $\mathrm{THz}$ frequency range, such effects will be weaker but may still account for some of the observed discrepancy. The scattering rates reported here are indeed much closer to theoretical predictions than those obtained from optical reflectivity measurements. 6,7

While charge-charge interaction affects the operation of 
many electronic devices under high current density, ${ }^{1}$ we show here that these interactions are significantly weaker in polar semiconductors such as $\mathrm{TiO}_{2}$ compared to $\mathrm{Si}$. In the density range accessed here, electron-hole scattering is expected to behave as $1 / \tau_{e h} \propto N_{0} /\left(\varepsilon^{2} \sqrt{\mu}\right),{ }^{5}$ where $\mu$ is the electron-hole reduced mass. Both $\varepsilon$ and $\mu$ are more than an order of magnitude higher in $\mathrm{TiO}_{2}$ (Ref. 9) than in $\mathrm{Si}^{18}$ so that, at low temperature, electron-hole scattering is expected to be important for densities around 3 orders of magnitude smaller in $\mathrm{Si}$ than in $\mathrm{TiO}_{2}$, in agreement with our observations. As a result, these materials will behave very differently in electro-optic devices, and density-dependent effects will play a role already at relatively low densities in nonpolar semiconductors. This is exemplified by the highly nonlinear density response of silicon based solar cells, where electronhole scattering can play a significant role in device operation, especially under strong illumination conditions. ${ }^{2}$

This work is part of the research program of the "Stichting voor Fundamenteel Onderzoek der Materie (FOM)," which is financially supported by the "Nederlandse Organisatie voor Wetenschappelijk Onderzoek (NWO)." We are grateful to H. G. Muller for helpful discussions.
*FAX: +44 1392 264111; e.hendry@exeter.ac.uk

${ }^{1}$ T. T. Mnatsakanov, M. E. Levinshtein, A. G. Tandoev, S. N. Yurkov, and J. W. Palmour, J. Appl. Phys. 97, 103715 (2005).

${ }^{2}$ T. T. Mnatsakanov, V. B. Shuman, L. I. Pomortseva, D. Schroder, and A. Schlogl, Solid-State Electron. 44, 383 (2000).

${ }^{3}$ H. M. von Driel, Appl. Phys. Lett. 44, 617 (1984).

${ }^{4}$ B. E. Sernelius, Phys. Rev. B 40, 12438 (1989).

${ }^{5}$ M. Combescot and R. Combescot, Phys. Rev. B 35, 7986 (1987).

${ }^{6}$ D. Hulin, M. Combescot, J. Bok, A. Migus, J. Y. Vinet, and A. Antonetti, Phys. Rev. Lett. 52, 1998 (1984).

${ }^{7}$ K. Sokolowski-Tinten and D. von der Linde, Phys. Rev. B 61, 2643 (2000).

${ }^{8}$ E. Hendry, J. M. Schins, L. P. Candeias, L. D. A. Siebbeles, and M. Bonn, Phys. Rev. Lett. 92, 196601 (2004).

${ }^{9}$ E. Hendry, F. Wang, J. Shan, T. F. Heinz, and M. Bonn, Phys. Rev. B 69, 081101(R) (2004).

${ }^{10}$ A. Nahata, A. S. Weling, and T. F. Heinz, Appl. Phys. Lett. 69, 2321 (1996).

${ }^{11}$ T. I. Jeon and D. Grischkowsky, Phys. Rev. Lett. 78, 1106 (1997).

${ }^{12}$ N. Saeta, J. F. Federici, B. I. Greene, and D. R. Dykaa, Appl. Phys. Lett. 60, 1477 (1992).

${ }^{13}$ T. Sjodin, H. Petek, and H. L. Dai, Phys. Rev. Lett. 81, 5664 (1998).

${ }^{14}$ Since the plasma is not confined to the surface of the semiconductor, we expect it to behave as a three dimensional, and not a two dimensional, plasma. Indeed, charge diffusion during the $10 \mathrm{ps}$ between the excitation and $\mathrm{THz}$ probe pulses will lead to an effective charge penetration depth larger than that for the excitation light and comparable to the electron mean free path. For $\mathrm{Si}$, the electron diffusion length for $10 \mathrm{ps}$ is $\sim 200 \mathrm{~nm}$, much larger than the optical penetration of $\sim 10 \mathrm{~nm}$. Therefore, the magnitudes of $\sigma^{a v}$ extracted using Eq. (1) are only approximate for $\mathrm{Si}$. In $\mathrm{TiO}_{2}$, the diffusion length is significantly lower, and neglecting diffusion is justified.
${ }^{15}$ M. Cardona and G. Harbeke, Phys. Rev. 137, A1467 (1965).

${ }^{16}$ G. E. Jellison and F. A. Modine, Appl. Phys. Lett. 41, 180 (1982).

${ }^{17}$ H. K. Nienhuys and V. Sundstrom, Appl. Phys. Lett. 87, 012101 (2005).

${ }^{18}$ C. Jacoboni, C. Canali, G. Ottaviani, and A. A. Quaranta, SolidState Electron. 20, 77 (1977).

${ }^{19}$ The spatial decay of the excitation density is given by $N(x)$ $=f_{l} / l_{0} \exp \left(-x / l_{0}\right) T$, where $T$ and $l_{0}$ are the sample interface transmission coefficient (at $x=0$ ) and the penetration depth of the excitation light, respectively (Refs. 13 and 14). We report the average excitation density $N^{a v}=\left(1 / l_{0}\right) \int_{0}^{l_{0}} N(x) d x \approx 0.63\left(f_{l} / l_{0}\right) T$, in the region from $x=0$ to $l_{0}$.

${ }^{20} \mathrm{We}$ introduce an approximation with Eq. (1) by assuming a homogenous excitation region. For Drude model conductivity, the exact response is $\Delta E(\omega) / E(\omega)=-\left(l_{0} / 2 \varepsilon_{0} c \sqrt{\varepsilon} L\right) \int_{0}^{L}\left[e^{2} N(x) /\right.$ $\left.m_{\text {con }}\right]\{\tau(x) d x /[1-i \omega \tau(x)]\}$, where $L$ is the crystal thickness. By comparing the results of this equation to those of Eq. (1), we estimate that mean electron scattering rates $\left\{\int_{0}^{\infty}[N(x) /\right.$ $\left.\tau(x)] d x / \int_{0}^{l_{0}} N(x) d x\right\}$ only differ significantly (approaching 20\%) from $1 / \tau^{a v}$ for the highest fluences used.

${ }^{21}$ D. M. Riffe, J. Opt. Soc. Am. B 19, 1092 (2002).

${ }^{22}$ D. L. Young, T. J. Coutts, V. I. Kaydanov, A. S. Gilmore, and W. P. Mulligan, J. Vac. Sci. Technol. A 18, 2978 (2000).

${ }^{23}$ D. Long and J. Myers, Phys. Rev. 115, 1107 (1959).

${ }^{24}$ Band filling leads to a Fermi electron (or hole) velocity, given by $v_{f}=\hbar / m_{\text {DOS }}\left(3 \pi^{2} N_{0} / M\right)^{1 / 3}$ for $M$ equivalent band minima and the density of states effective mass $m_{\text {DOS }}$. The electron-phonon scattering rate is then approximately described by $1 / \tau_{p h}=1 / \tau_{0}$ $+v_{f} / l_{p h}$, where $l_{p h}$ is the electron mean free path. For acoustic phonon scattering, $l_{p h}$ is independent of $v_{f}$ [see J. Bardeen and W. Shockley, Phys. Rev. 80, 72 (1950)] and can be estimated from $l_{p h}=\tau_{0} \sqrt{3 k_{b} T / m_{\text {DOS }}}$.

${ }^{25}$ K. M. Glassford and J. R. Chelikowsky, Phys. Rev. B 46, 1284 (1992).

${ }^{26}$ B. E. Sernelius, Phys. Rev. B 43, 7136 (1991). 